\title{
Research on the diagnosis of peritoneal micrometastases in tumors of the subdiaphragmatic digestive tract
}

\author{
Department of Surgery, Faculty of Medicine, University "Ovidius" Constanta, Romania
}

\begin{abstract}
:
This presentation is aimed at analyzing the possibility of detecting the peritoneal micrometastases through multiple peritoneal biopsies to the patients with tumors of the subdiaphragmatic digestive tract.

Nowadays, detection of the micrometastases is performed indirectly through cytological analysis and / PCR analysis of the peritoneal liquid, through the histopathological study of the peritoneal fragments intraoperatively collected and the immunohistochemical study.

This presentation is aimed at analyzing the possibility of detecting the peritoneal micrometastases through multiple peritoneal biopsies and to correlate this with various factors such us: staging neoplasia, the affected organ, histopathological type, tumoral grading.

Inclusion of the patients that find themselves in the early stage of peritoneal carcinomatosis - of peritoneal micrometastases - would allow for the application of an adequate treatment and could greatly increase the patient's survival chances.
\end{abstract}

Key words: micrometastasis, peritoneal lavage, immunohistochemistry, histopathological study, peritoneal carcinomatosis, cytoreductive surgery, intraperitoneal chemohyperthermia.

Ciufu Nicolae

University „Ovidius”, Faculty of Medicine, Constanta

1, Aleea Universitatii

Tel/Fax: +40-241-605-004

\section{Introduction}

The peritoneal carcinomatosis has always been regarded as a terminal condition. It is present in 10$30 \%$ of the patients that suffer from gastrointestinal cancer during the initial surgical intervention and at the same time it constitutes a frequently met condition on patients that develop recurrent neoplasias. Metastases are defined in the oncology treatises as the transfer of an affection to an organ (or a part of it) from another organ to which the first one is directly linked (connected).[6]

This colonization having a starting point in the primary tumor is closely related to the properties of the tumor cells. Tumor cells can migrate not only through the penetration of the lymphatic or blood vessels but also through that of the peritoneal cavity. Neoplastic cells have their own mobility and rapid division creates an increased local pressure, followed by cell detachments. Following observation from our many decades practice, they have been known as an invasion of the viscera serous membrane by the abdominal malignant tumors, that which leads rapidly to ascites and carcinomatosis.

The cases of pelvic inseminations that have their starting point at the level of gastric cancers (Krukenberg tumors) are very well known.

The individual mobility of the cells has different degrees that go in parallel with the severity of the neoplastic disease. Enzymes guilty for "transporting" these cells through the tissues in proximity are also known. Finally, the results are small cell deposits in the lymph nodes, in various organs or cavities, such as the peritoneal or pleural one. Their existence is not disputed by anyone. 
The presence of cancer cells in the ascitic liquid or in the pleural liquid already represents an undeniable truth. More or less known is the presence of cell inseminations at the level of the parietal and visceral peritoneum. These micrometastases are responsible for the peritoneal carcinomatosis identified after a short interval in the case of the "ideal" extirpation of the intra-abdominal tumors.

Numerous studies of the natural history of the disease have shown that for this group of patients, the average survival rate is of 6 months. [3]

In the last decade, a new type of locoregional therapy, that associates cytoreductive surgery and intraperitoneal chemohyperthermia, reported promising results $[4,5]$, but due to limited benefits, the increased morbidity and mortality rates and very high costs, this management plan requires an appropriate selection of the patients.

Patients' selection is extremely important in optimizing the treatment's results and allows at the same time the exclusion of those that would not receive benefits from a life-threatening therapy associated with a high morbidity rate. In establishing the eligibility of the patients for the combined treatment, it is necessary to use quantitative indicators of prognosis; these indicators represent guidelines for the selection of various therapy methods and are aimed maximizing the treatment's benefits and at excluding the patients that would receive minimal benefits.

\section{Patients and Method}

In the clinical study were included 392 patients from 1404 patients operated in General Surgery Department of the Constanta County Emergency Hospital during the period Ianuary 2003- December 2009. Patients included in the study had been diagnosed with neoplasm of the subdiaphragmatic digestive tract.

The criteria for the inclusion in the study were the following:
- absence of direct and indirect imaging data proving the presence of intraperitoneal secondary determinations, before the surgery to the patients diagnosed with tumors of subdiaphragmatic digestive tract. The diagnosis was confirmed by endoscopic biopsy and/or histopathlogical examination.

- absence of peritoneal metastases macroscopically visible on laparotomy.

All patients were submitted to cytology of the peritoneal lavage liquid, histopathological examination of the peritoneum fragments harvested from 6 sites (the right and left subdiaphragmatic peritoneum, the greater omentum, the right lateral and left lateral paracolic gutters, the pelvis) and to immunohistochemical examination. The immunohistochemical examination has not been carried out to the 47 patients, where the histopathological examination showed the inclusion and fixing defects

Diagnosis of the peritoneal micrometastases is essential for the appropriate selection of patients and for the correct establishment of an adequate treatment. Below we will provide an overview of the three diagnosis methods of these micrometastases.

The first diagnosis method of the peritoneal micrometastases is represented by the cytological study and PCR study of the peritoneal lavage.

The peritoneal liquid is obtained through lavage performed immediately after laparotomy, before manipulating the intraperitoneal tumor/ tumors. In this respect, $500 \mathrm{ml}$ of $0,9 \%$ physiological serum is instilled in the peritoneal cavity and is evenly distributed through gentle maneuvers. After that, 3 samples of $30 \mathrm{ml}$ each of peritoneal liquid from the right hypchondrium, from the left hypchondrium and from the Douglas cul-de-sac are harvested. After that it is fixed with a $33 \%$ methanol solution, then centrifuged for $10 \mathrm{~min}$, the obtained cell concentrates are re-fixed with methanol solutions, they are placed on the slides, they are stained and they are studied on the microscope. [1]

The cytology of the peritoneal lavage liquid during surgery or that of second look laparoscopy for carcinomas is a fundamental investigation in detecting the peritoneal microstases. It is important to avoid obtaining false-positive or false-negative results through an adequate approach of the patients. 
In benign cases, a rate of $4,5 \%$ false-positive results has been reported. Hyperplasia of the mesothelioma associated with the calcified structures can also be the cause of confusion, as it mimics serous neoplasms with psammoma bodies [1]. It is usually found on specimens obtained from patients with pelvic inflammatory disease.

Real-time quantitative RT-PCR (reverse transcription polymerase chain reaction) of the peritoneal liquid through tumor markers such as CEA, CK20, has obtained results that are superior to cytology, but the method is still being evaluated. [1]

The second method of diagnosis of the peritoneal micrometastases is represented by the histopathological study of peritoneal biopsies.

Peritoneal biopsies are harvested from 6 sites:

- the right and left subdiaphragmatic peritoneum;

- the greater omentum;

- the right and left paracolic gutters;

- the pelvis.

The dimensions of the parts harvested are of 1-2 cm minimum.

The material harvested during surgical intervention is sent to the pathological anatomy laboratory, where it is processed in order to be examined on the microscope.

After having harvested it, the biological material is fixed in a neutral formalin solution $10 \%$ for 48-72 hours, at laboratory temperature. The fixating $10 \%$ formalin solution has been obtained from formaldehyde (commercial formalin 40\%) as follows:

- commercial formalin

1 part

- distilled water

9 parts.

Calcium bicarbonate has been added to the solution in order to reach neutral $\mathrm{pH}$ to neutralize the formic acid, formed spontaneously in concentrated solutions.

Formalin is a cheap fixing solution, with high power of penetration in the biological material, it forms little few precipitates, it does not produce modifications of the color and of the structure of the preparations, it allows for the achievement of a greater number of stains and it is less toxic for the people that use it.

In order to achieve a good fixation of the entire harvested material, we have taken steps to have a fixing solution volume of 15-20 times larger than that of the volume of the piece to be fixated, thus the biological material has been placed in glass jars of approximately $800-2000 \mathrm{ml}$ each according to the size of the part harvested in the operating room. In order to have the entire piece penetrated in a homogenous way by the fixing solution, on the bottom of the containers with fixing solution we have placed plastic grids on top of which we put filter paper, so that even the part of the biological material in contact with glass container comes in direct contact with the fixing solution. The fixing process of the biological material is an essential operation in the histopathological an immunohistochemical studies, without which one cannot obtain good quality histological preparations.

\section{Objectives:}

- to freeze the vital processes from within the cells and the tissues in a certain biological state;

- to prevent the process of alteration of the cell and tissue structures extracted from the organism by prevention of the autolysis;

- to prevent structural alterations caused by a bacterial attack;

- to prepare the cells and tissues for an adequate staining.

A good fixating solution must meet the following requirements:

- it must keep the biological tissues structures to be fixated as close as possible to the "in vivo" state, it must not modify the reports between the various tissue components so that it does not produce artifacts;

- to offer a certain consistency of the biological material, which is necessary for the sectioning and following processing;

- to have a high penetration power in the harvested biological material;

- to be devoid of toxicity for the ones using it;

- to evidentiate the histological structures through the desired staining technique; 
- to be in sufficient quantity (its volume must be of 10-20 times larger than the volume of the piece to be fixated).

The wide use of formaldehyde for fixation has many advantages: a reduced cost, it does not harden excessively the pieces, it allows for the achievement of a great number of histological stains, it modifies (contracts) the pieces very little, it penetrates and preserves well the blood and adipose tissue, normal colors are preserved better than in the case of other fixating solutions, it achieves a better fixation of the proteins, by stopping this way the loss of glycogen from the tissue in aqueous solutions.

Because formalin $10 \%$ is a gentle fixating solution, the tissue fragments can be kept for longer periods of time without the cellular or intracellular structures getting altered.

Formalin fixates the tissues, through a main reaction with proteins. It establishes connections with 11 groups of chemical reactive terminals of the proteins, except for the acid groups. The majority of the bridges or connections are weak and reversible through washing. The connections are formed with the amino, imino, guanidyl, amido, peptidyl, hydroxyl, carboxyl, sulphydryl groups and aromatic groups. Irreversible reactions are achieved with thyroxine, tryptophan, phenylalanine and histidine.

Through exposureto air, formaldehyde oxidizes, forming gradually formic acid, even in the diluted unbuffered solution. The acid $\mathrm{pH}$ has a destructive effect on the tissues undergoing fixation. During the fixation process in the acid solution, through the destruction of the red blood cells, a blackish pigment (haematin) results following the reaction between the formic acid and the obtained hemoglobin. Fixating the blood rich tissues in formalin solution with a $\mathrm{pH}-5$ leads to the formation of a great quantity of pigment due to the lysis of the erythrocytes. The pigment is of black-brown color, microcrystalline and it forms numerous artifacts, preventing the achievement of a correct histopathological diagnosis. It can be dissolved and removed in alkaline solutions or through a process of treatment with an alcohol picric acid solution (10-12 gr. picric acid is dissolved in $100 \mathrm{ml}$ of ethylic alcohol). After being fixated, the biological material was washed with tap water for 24 hours, in order to remove the formalin from the tissues and then it was included in paraffin.

The utilized histological technique has the following stages:

- dehydration,

- clarification,

- paraffin processing,

- the actual inclusion,

- the sectioning of the block,

- the placement of the sections on the slides, followed by their drying,

- staining the sections,

- interpretation.[4]

The third method of diagnosis of the peritoneal micrometastases is represented by the immunohistochemical study of peritoneal microstases.

The studied biological material is represented by peritoneum fragments which have been harvested on the occasion of the surgical treatment applied for curative purposes, with the exclusion of the cases in which, after an ordinary histopathological examination, inclusion or fixation defects have been present and they would have led to erroneous results. [4]

A thorough selection and processing technique is applied to the biological material, because an inadequate fixation process can determine an alteration of the obtained results due to the their inactivation or structural alteration.

Immunohistochemical methods used:

From the selected blocks included on paraffin, sections of 4 microns thickness are cut with the aid of a microtome, then the sections have initially been deparaffinized through three successive baths of xylene and rehydrated by being washed in decreasing strengths of alcohol $(100 \%, 70 \%, 50 \%, 15$ minutes each).

In the end, the sections have been bathed in distilled water to completely remove the traces of alcohol.

The first stage of the immunohistochemical technique resides in the antigen recovery by boiling in the microwave in a substance chosen depending on the antibody.

The second stage has been represented by the incubation with a solution of hydrogen peroxide $1 \%$ in distilled water for 30 minutes. 
The third stage has been represented by the incubation of the sections in a bovine serum albumin $1 \%$ for 3 minutes.

After this stage, without washing, after removing the blocking solution, the main antibody has been added to the slide and after that the biological material has been incubated in the fridge at 4 degrees Celsius.

For the reconstitution solution of the antibody, bovine serum albumin $1 \%$ is being used.

The next stage is represented by the addition on the section of the secondary detection system, a complex secondary-antibody primary-antibody that is bound by a polymer with numerous peroxidase molecules (the ENVISION, TAKO system). [4]

The final stage of the immunohistochemical reaction was the actual detection of the signal.

By using the interpretation criteria from the specialty literature, the intensity of the staining is marked as follows:

-+++ intensely positive staining, over $80 \%$ of the cells with diffuse distribution, clearly visible on examination with a low magnification lens,

-++ moderate intensity staining, at 30-80\% of tumor cells with focal adhesion, well visible on examination with medium magnification lens,

-+ weak intensity staining, 5-30\% isolated cells, well visible on examination with a low magnification lens,

$-+/$ - reduced intensity staining, at less than 5\% of tumor cells,

- - staining absence.

\section{Results and Discussions:}

The cytological examination of the peritoneal lavage liquid was positive for the malignant cells in 67 cases of the $392(17.1 \%)$. In cases with gastrointestinal carcinoma the percent was $23.53 \%$ (16 of the 68 cases), in cases with colo-rectal carcinoma the percent was $15.74 \%(51 / 324)$, the difference was statistically significant (0.05). Sensitivity and specificity of the cytological examination of the peritoneal lavage liquid was $59 \%$ and $96 \%$. The positive predictive value was 0.47 and the negative predictive value was 0.98 . There was no recorded false positive examination in the control group.

The association of the positive peritoneal lavage with following features was statistically significant.

- tumoral gradingG3 $(\mathrm{p}=0.003)$

- the invasion of de serosa $(\mathrm{p}=0.03)$

- tumoral invasion (0.01)

- tumoral necrosis (0.03).

Table I. Corellations between the cytology of the peritoneal lavage liquid and clinical-pathological parameters

\begin{tabular}{|c|c|c|c|c|}
\hline Variable & $\begin{array}{c}\text { Positive } \\
\text { (nr) }\end{array}$ & $\begin{array}{l}\text { Total } \\
(\mathrm{nr})\end{array}$ & $\begin{array}{c}\text { Positive } \\
(\%)\end{array}$ & $\begin{array}{c}\text { Value } \\
P\end{array}$ \\
\hline Sex & & & & .38 \\
\hline Male & 46 & 220 & 20,91 & \\
\hline Female & 21 & 172 & 12,21 & \\
\hline Age & & & & .71 \\
\hline$<40$ years & 17 & 114 & 14,91 & \\
\hline$>40$ years & 50 & 278 & 17,99 & \\
\hline \multicolumn{4}{|l|}{$\begin{array}{l}\text { Localization of the } \\
\text { primary tumor }\end{array}$} & .05 \\
\hline Stomach, small bowel & 16 & 68 & 23,53 & \\
\hline Colon and rectum & 51 & 324 & 15,7 & \\
\hline Size of the tumor & & & & .53 \\
\hline$<5 \mathrm{~cm}$ & 15 & 136 & 11,03 & \\
\hline$>5 \mathrm{~cm}$ & 52 & 256 & 20,31 & \\
\hline \multicolumn{4}{|c|}{ Mucinos character of the tumor } & .26 \\
\hline Yes & 26 & 83 & 31,33 & \\
\hline No & 41 & 309 & 13,27 & \\
\hline Vascular invasion & & & & .15 \\
\hline Yes & 18 & 67 & 26,87 & \\
\hline No & 49 & 325 & 15,08 & \\
\hline Neural invasion & & & & .76 \\
\hline Yes & 9 & 56 & 16,07 & \\
\hline No & 58 & 336 & 17,26 & \\
\hline \multicolumn{4}{|c|}{ The invasion of the serosa } & .03 \\
\hline Yes & 60 & 276 & 21,74 & \\
\hline No & 7 & 116 & 6,03 & \\
\hline \multicolumn{4}{|l|}{ Local adenopathy } & .59 \\
\hline Yes & 29 & 261 & 11,11 & \\
\hline No & 38 & 131 & 29,01 & \\
\hline \multicolumn{4}{|l|}{ Tumoral invasion } & .01 \\
\hline $\mathrm{T} 1-\mathrm{T} 2$ & 4 & 70 & 5,71 & \\
\hline T3-T4 & 63 & 322 & 19,57 & \\
\hline \multicolumn{4}{|l|}{ Tumoral necrosis } & .03 \\
\hline Yes & 23 & 94 & 24,47 & \\
\hline No & 44 & 298 & 14,77 & \\
\hline \multicolumn{4}{|l|}{ Tumoral grading } & .003 \\
\hline G1-G2 & 8 & 245 & 3,27 & \\
\hline G3 & 59 & 147 & 40,14 & \\
\hline
\end{tabular}


The histopathological study of the peritoneal biopsies was positive for the malignant cells in 62 cases of the 392.(15.8\%) In cases with gastrointestinal carcinoma the percent was $22.06 \%$ (15 from 68 cases). In cases with colo-rectal carcinoma the percent was $14.55 \%(47 / 324)$. The difference was statistically significant $(0.05)$.

The association of the peritoneal biopsy with the following features was statistically significant.

- tumoral grading G3 ( $\mathrm{p}=0.01$ )

- the invasion of the serosa $(\mathrm{p}=0 ., 02)$

- tumoral invasion (0.02)

- tumoral necrosis $(0.03)$.

Table II. Corellations between peritoneal biopsia and clinical-pathological parameters.

\begin{tabular}{|c|c|c|c|c|}
\hline Variable & $\begin{array}{l}\text { Pozitiv } \\
\text { (nr) }\end{array}$ & $\begin{array}{c}\text { Total } \\
\text { (nr) }\end{array}$ & $\begin{array}{c}\text { Pozitiv } \\
(\%)\end{array}$ & $\begin{array}{c}\text { Value } \\
\mathrm{P}\end{array}$ \\
\hline Sex & & & & .54 \\
\hline Male & 43 & 220 & 19,55 & \\
\hline Female & 19 & 172 & 11,05 & \\
\hline Age & & & & .84 \\
\hline$<40$ years & 17 & 114 & 14,91 & \\
\hline$>40$ years & 45 & 278 & 16,19 & \\
\hline $\begin{array}{l}\text { The localisation of the } \\
\text { primary tumor }\end{array}$ & & & & .05 \\
\hline Stomach, small bowel & 15 & 68 & 22,06 & \\
\hline Colon and rectum & 47 & 324 & 14,51 & \\
\hline Size of de tumor & & & & .12 \\
\hline$<5 \mathrm{~cm}$ & 13 & 136 & 9,56 & \\
\hline$>5 \mathrm{~cm}$ & 49 & 256 & 19,14 & \\
\hline $\begin{array}{l}\text { The mucinous character } \\
\text { of the primary tumor }\end{array}$ & & & & .09 \\
\hline Yes & 23 & 83 & 27,71 & \\
\hline No & 39 & 309 & 12,62 & \\
\hline Vascular invasion & & & & .11 \\
\hline Yes & 16 & 67 & 23,88 & \\
\hline No & 46 & 325 & 14,15 & \\
\hline Neural invasion & & & & .69 \\
\hline Yes & 8 & 56 & 14,29 & \\
\hline No & 54 & 336 & 16,07 & \\
\hline The invasion of the serosa & & & & .02 \\
\hline Yes & 56 & 276 & 20,29 & \\
\hline No & 6 & 116 & 5,17 & \\
\hline Local adenopathy & & & & .21 \\
\hline Yes & 37 & 261 & 14,18 & \\
\hline No & 35 & 131 & 26,72 & \\
\hline Tumoral invasion & & & & .02 \\
\hline T1-T2 & 3 & 70 & 4,29 & \\
\hline T3-T4 & 59 & 322 & 18,32 & \\
\hline Tumoral necrosis & & & & .03 \\
\hline Yes & 21 & 94 & 22,34 & \\
\hline No & 41 & 298 & 13,76 & \\
\hline Tumoral grading & & & & .01 \\
\hline G1-G2 & 7 & 245 & 2,86 & \\
\hline G3 & 55 & 147 & 37,41 & \\
\hline
\end{tabular}

\section{Eonclusions}

1.The early diagnosis of peritoneal carcinomatosis, at its micrometastasis stage, can represent the key to a significant improvement of its therapeutic results. It can also contribute to a correct staging of the neoplasia during surgical intervention, by avoiding substaging and indicating a more aggressive treatment of the neoplastic disease.

2.In the diagnosis of peritoneal micrometastases, the peritoneal biopsy has obtained results similar to those of cytological examination of the peritoneal lavage.

3.The histopathological study of peritoneal metastases has demonstrated the fact that the largest number of tumors is represented by moderately differentiated adenocarcinomas that have their origin in the mucous membrane of the subdiaphragmatic digestive tract.

4. The immunohistochemical study of peritoneal tumors is extremely useful in the establishment of the origin of the primary tumor using specific antibodies. The immunohistochemical examination evaluates the proliferative activity of the tumors and tumor angiogenesis.

\section{References}

1. Dalal K.M., Woo Y. \& Kelly K. (2008). Detection of micrometastases in peritoneal wasing of gastric canser patient by the reverse transcriptase polymerase chain reaction, Gastric cancer . 20613

2. Sirma T. (Ph.D.), Voinea F. (scientific advisor). (2009). The etiology of peritoneal carcinomatosis. Thesis, University "Ovidius" of Constanta, Romania

3. Raptopoulos V. \& Groutsoyannas N. (2001). Peritoneal Carcinomatosis. Eur radio. 11:21952206 
4. Pecciarinii L., Cangi M.D. (2001). Identifying the primary site of metastatic carcinoma; the increasing role of immunohistochemistry. Curr diag pathol. 7: 168-175

5. Sugarbaker PH.., Schellinx M.E. \& Chang D. (1996). Peritoneal carcinomatosis from adenocarcinoma of the colon. World J Surg. .20: 585-592
6. Goldstein P. et al. (2005). Management of peritoneal carcinomatosis from colon cancer, gastric cancer and appendix malignancy. Cancer theraphy. 3: 299-320. 